CAHIERS DE

NARRATOLOGIE

\section{Cahiers de Narratologie}

Analyse et théorie narratives

$10.1 \mid 2001$

La voix narrative

\title{
Le « je » polyphonique du monologue intérieur dans L'Etranger de Camus.
}

\section{Cvetanka Conkinska}

\section{(2) OpenEdition}

1 Journals

\section{Electronic version}

URL: http://journals.openedition.org/narratologie/6954

DOI: 10.4000/narratologie.6954

ISSN: 1765-307X

\section{Publisher}

LIRCES

\section{Printed version}

Date of publication: 1 January 2001

Number of pages: $285-295$

ISBN: 2914561032

ISSN: 0993-8516

\section{Electronic reference}

Cvetanka Conkinska, "Le « je » polyphonique du monologue intérieur dans L'Etranger de Camus.",

Cahiers de Narratologie [Online], 10.1 | 2001, Online since 27 November 2014, connection on 23

February 2021. URL: http://journals.openedition.org/narratologie/6954 ; DOI: https://doi.org/10.4000/ narratologie.6954 


\title{
LE « JE » POLYPHONIQUE DU MONOLOGUE INTÉRIEUR DANS L'ETRANGER D'ALBERT CAMUS
}

\author{
Cvetanka CONKINSKA \\ Université de Nice-Sophia Antipolis
}

La Voix Narrative répond aux questions telles que : Qui raconte ? Qui est le Narrateur? Quel est le degré de son implication dans l'Histoire qu'il raconte ? Passe-t-il de NarrateurNarrant en Narrateur-Narré ? Y a-t-il une polyphonie provoquant des changements considérables dans le niveau narratif?

La Voix Narrative étudie donc la relation entre l'Histoire et le Narrateur. Le Narrateur initial raconte l'Histoire et, très souvent, laissant sa parole aux Personnages, il fait apparaître de nouveaux Narrateurs. Parfois, un même Narrateur sert de porte-parole aux Personnages, multipliant ainsi les voix narratives et provoquant une Polyphonie. Genette et Todorov ont longuement étudié les techniques de cette voix qui, par ailleurs, nous aide à découvrir le texte dans une autre dimension, plus approfondie. Ou alors, il faudra préciser que cela dépend de la complexité du texte narratif, car, il y a des textes «insolvables » et restant toujours aussi compliqués après qu'avant une analyse. C'est, peut-être, le cas de L'Etranger d'Albert Camus; plusieurs critiques l'ont " sélectionné » comme étant l'un des livres des plus ambigus, car l'ambiguïté l'accompagne à tout point de vue : forme, thème, idée, personnage ; la discipline de la Narratologie le mentionne aussi par son ambiguïté :

«Le statut narratif de L'Etranger [...] se trouve être d'emblée un des exemples les plus frappants pour ce genre de discussion puisqu'il consiste en une description 
apparemment objective, "béhavioriste », écrite à la première personne $» .1$

En 1960, Fitch évoque le problème « des nombreuses interprétations du roman ${ }^{2}$. Il paraît même que cette ambiguïté est « voulue et recherchée » par Camus ${ }^{3}$.

Le récit de L'Etranger est fait à la première personne, sous forme de monologue intérieur, ce qui ferait également penser à un journal intime. Vu la forme du texte, l'ambiguïté s'installe déjà, car les deux formes sont fort possibles.

L'ambiguïté de ce livre reste-t-elle d'actualité ? Aujourd'hui encore, on peut dire, comme cela a été dit par Jean-Claude Brisville en 1959 : " une vérité ambiguë brûle dans la clarté de cette œuvre, sans doute une des plus mystérieuses de notre temps ». ${ }^{4}$ Cela dit, notre étude ne sera qu'une interprétation personnelle de plus, traitant de l'analyse narratologique, et dont le but sera d'attribuer davantage à la connaissance de ce roman mystérieux, voire énigmatique.

Mais, que représente L'Etranger en langue narratologique? Et sa Voix Narrative?

On parle d'un roman « béhavioriste » qui, par définition, est un récit extra-hétérodiégétique à focalisation externe. Donc, récit raconté à la troisième personne. Or, L'Etranger est, à quelques exceptions près, un récit béhavioriste, mais raconté à l'impossible première personne du singulier. On sait qu'un Je-Narrateur sous-entend une focalisation interne et un récit intra-homodiégétique, voire autodiégétique; or, ce n'est guère le cas avec L'Etranger. Au contraire même, d'où toute la complexité dont «bénéficie » L'Etranger. D'où sa singularité aussi.

${ }^{1}$ Dominique RABATÉ : Vers une littérature de l'épuisement, Paris, éd. José Corti, 1991, p. 86.

2 Brian T. FITCH : Narrateur et Narration dans l'Etranger de Camus, analyse d'un fait littéraire, Lettres Modernes, 1960 (deuxième édition revue et augmentée en 1968), pp. 1-2.

3 Op. cit., p. 5.

4 Jean-Claude BRISVILLE : Camus, Paris. Gallimard, 1959, p. 53. 
Sur le plan des personnages, au fur et à mesure que le texte avance, il y a un effet inversement proportionnel. Dans la première partie, les personnages sont nommés et vivent au jour le jour: Meursault, Marie, Raymond, etc.; dans la deuxième partie, les personnages anonymes incarnent des personnes : avocat, juge, juré, accusé, etc. «Meursault est un personnage unique dans son genre et - dit Bernard Pingaud - semble se garde[r] de tout jugement et semble vouloir limiter son ambition à un simple témoignage $»^{5}$. Mais pas tout au long du récit, car plus on avance dans le texte, plus le personnage de Meursault semble se sensibiliser et sortir de l'absurde anesthésiant. Voici quelques extraits de L'Etranger pris dans l'édition Gallimard - Folio de 1942 et où l'on remarque les verbes de subjectivité et de jugement annonçant la fin de l'absence de sentiment chez Meursault :

« Moi, j'étais lassé de répéter ainsi la même histoire et $i l$ me semblait que je n'avais jamais autant parlé » p. 105.

« Cette déclaration a paru faire un effet considérable Mais lui-même paraissait ébranlé.... » p. 148.

«Le fond de sa pensée [celle du procureur], si j’ai bien compris, c'est que j'avais prémédité mon crime.» p. 152 .

Maintenant, Meursault-Narrateur semble fouiller dans le fond de la pensée de quelqu'un d'autre, alors qu'avant, il n'était pas capable de connaître sa propre pensée. Avec le dernier exemple, nous ne pouvons pas nous empêcher de citer Rabatel lorsqu'il parle de Personnage devenant Narrateur d'un récit enchâssé et dit ceci : "Sur le plan linguistique, l'accès aux pensées d'autrui est permis à tout locuteur qui utilise les modalités du discours rapporté ou transposé ou narrativisé, aucune de ces structures n'étant a priori réservée au Narrateur ou au Personnage ${ }^{6}$. Dans notre cas, Meursault Personnage et Narrateur et qui utilise tous les discours dont parle Rabatel, déstabilise, une fois encore, la théorie béhavioriste : en plus de l'emploi du «je » autodiégétique pour

5 Bernard PINGAUD commente L'Etranger, Folio Gallimard, 1992, p. 79.

6 Alain RABATEL: La construction textuelle du point de vue, Delachaux et Niestlé S.A., Lausanne (Suisse) - Paris, 1998, p. 151. 
raconter une histoire extra-hétérodiégétique, il s'introduit dans une focalisation interne, voire omnisciente, confirmant ainsi un antibéhaviorisme.

Il faudra également évoquer la confusion entre Narrateur et Héros, laquelle confusion s'installe incontestablement lors d'un récit raconté à la première personne : L'Etranger en est un exemple par excellence ! En tant que récit à la première personne, la logique des choses dirait qu'il s'agit d'une autobiographie. Mais, Camus ne cède guère à cette logique. Son roman est tout sauf autobiographie. En ce sens, et si l'on prend en considération l'opinion de Todorov pour qui le récit à la première personne "n'explicite pas l'image de son narrateur, mais au contraire la rend plus implicite encore ", nous aurons une difficulté supplémentaire pour déchiffrer les Voix masquées derrière le « je » de Meursault. A première vue, ce « je » est responsable de tout ce qui se passe dans le texte narratif de L'Etranger: action ou plutôt non-action; narrateur initial et narrataire final ; narrateur dont les personnages deviennent des sujets-objets pour ses multiples micro-récits ; un je/personnage principal /narrateur à vision pourtant bien limitée, tel un observateur clinique ; un « je » qui narre en employant tout discours possible (discours direct, discours indirect, discours indirect libre, discours indirect narrativisé) tel un reporter des discours des autres, ou pour employer les termes de Genette, Meursault accomplit « rhésis et diégésis $\gg^{7}$; etc. Genette essaie de saisir Meursault en lui assignant « une focalisation interne avec paralipse presque totale des pensées. $»^{8}$ Meursault raconte ce qu'il fait et décrit ce qu'il perçoit, mais il ne dit pas s'il en pense quelque chose. Cependant, il cache un en lui un narrateur, un narrateur visible et présent, à la différence du narrateur initial dont on a l'habitude de dire qu'il est invisible. A certains moments, Meursault se dédouble à la fois en tant que narrateur visible et invisible.

7 Gérard GENETTE : Nouveau discours du récit, Editions du Seuil, 1983, p. 29.

8 Op. cit., p. 85. 
« Le Narrateur Initial, responsable de la totalité du récit dit Alain Rabatel - est sans aucun doute plus enclin à l'omniscience, en vertu de son statut que le narrateur second. Ce narrateur second peut se réduire à un « je » relativement désincarné... $»^{9}$ et passer ainsi en narrateur-personnage, puis en personnage-narrateur. Et « la profondeur de la perspective du personnage est limitée, puisque ce dernier est d'un niveau diégétiquement inférieur à celui du narrateur $»^{10}$, ce qui, en soi, annonce un changement de niveau narratif. Mais que faire lorsque Meursault est à la fois le scénariste, le metteur en scène, le cameraman et l'acteur ? Pourquoi est-il tout cela ? Que faire avec l'aliénation qu'il représente ? Et ce n'est pas tout, " qu'au surplus l'auteur fasse appel à des artifices de style - dit André Nicolas - et à l'utilisation monotone, par exemple, de la formule « il y a » jointe à un surprenant emploi des temps qui choque nos habitudes littéraires et nous entraîne plus impitoyablement dans cet envers de l'homme, et l'effet sur la conscience du lecteur, atteindra une sorte de paroxysme. Et c'est pourquoi Meursault est inoubliable ${ }^{11}$. Oui, inoubliable par sa complexité que nous pouvons imager ici par le fait d'avoir une maladie autre que ce que désignent ses symptômes; par son contraste aussi : l'apparaître fait naître le paraître, car le Meursault instinctif (presque bestial) du début du récit, évolue ensuite en un homme réfléchi. Suffisamment pour ne pas donner raison à l'absurde, et mourir dans l'absurde. Car Meursault meurt à cause de l'absurde, mais il ne meurt pas dans l'absurde. Ce point, apparemment thématique et non narratologique, pourrait, peut-être, expliquer la démarche grammaticale et stylistique de l'écrivain : ce dernier se serait servi du passé composé à la place du passé simple, pour se familiariser avec le langage monotone de la vie quotidienne qui mène à l'absurde, pour combattre cet absurde. D'ailleurs, souffrant des répétitions forcenées de travail monotone pendant son enfance, l'auteur

9 Alain RABATEL, La construction textuelle du point de vue, éd. Delachaux et Niestlé S.A. Lausanne - Paris, 1998, p. 146,

10 Op. cit., p. 149.

11 André NICOLAS : Camus, Paris, 1966 by Pierre Seghers Editeur, pp. 9-10. 
Camus fuyait tout travail répétitif et non créatif. C'est un moment implicite rare où l'on reconnaît Albert Camus dans $L^{\prime}$ Etranger ${ }^{12}$. Qui sait, ce fait est, peut-être, à l'origine même de ce héros anti- héros qu'est Meursault. L'auteur a donc pu se permettre de choquer avec ses formes verbales, à l'image de la nature de son personnage. Evoquant Meursault, Brisville dit ceci : «Parlant de lui, Meursault prend la voix d'un témoin qui, l'observant de l'extérieur, n'aurait que le souci de le décrire [...] [cela] attribue à l'habilité de l'auteur une technique qui est finalement justifiée par la nature même du héros ", lorsque par exemple, Meursault dit à son avocat que " ses besognes dérangent souvent ses sentiments » ou que " tout être souhaite un jour la mort de la personne qu'il aime le plus ».

12 L'autre moment implicite où l'on pourrait reconnaître le Camus auteur concret en auteur impliqué (selon les termes de LINTVELT Jaap dans ses Essais de Typologies Narratives, Paris, éd. José Corti, 1981, pp. 24-28) est lors des quelques répétitions du mot "maman " de la part de Meursault, à côté de "ma mère". Pour ceux qui connaissent l'immense affection silencieuse que Camus portait à sa mère, il ne serait pas difficile de deviner - et ce, malgré le contexte malheureux de L'Etranger qui, nous le soulignons une fois de plus, n'a rien d'autobiographique -, que l'on entend la voix de Camus lui-même dans le mot "maman". Ce sentiment affectueux de Meursault, par ailleurs unique ou presque, pourrait être interprété comme restant l'unique lien humain qui empêcherait le Meursault instinctif de s'abandonner entièrement à l'absurde, devenant, par la suite réfléchi et parlant même de la morale. Il reste à savoir, si Camus a vraiment voulu choquer en utilisant le mot "maman" pour un personnage tel que Meursault, ou si, simplement, cela lui échappait, inconsciemment, à quelques reprises, tellement son affection et son souvenir maternel (laquelle mère, soulignons-le, était toujours en vie au moment de la publication du roman) l'avait marqué, de sorte que, à chaque fois qu'il pensait "mère "-fût-ce un personnage ayant un mauvais rôle, telle la mère dans Le Malentendu -, il avait l'image de sa propre mère à qui il s'adressait toujours avec le terme "maman ». Ou encore un autre épisode authentique, à la page 167, lorsque Meursault Narrateur dit : "Je me suis souvenu dans ces moments d'une histoire que maman me racontait à propos de mon père. Je ne l'avais pas connu... " - tout cela est exact pour Camus auteur concret, tout comme l'histoire qui s'ensuit et qui relève de sa vie réelle. C'est la preuve aussi que, les quelques éléments relevant de la vie de l'écrivain n'empêchent pas le devenir d'un roman de fiction. 
L'imparfait et le passé composé dominent le récit, surtout dans la deuxième partie. "L'imparfait est - selon Alain Rabatel - bien une forme verbale propre à traduire la subjectivité des perceptions et leur valeur expériencielle/mémorielle »13. C'est exactement la fonction qu'a l'emploi de l'imparfait dans la seconde partie de L'Etranger : commenter, juger, réfléchir, se souvenir ; à la différence de cela, l'imparfait du début du récit sert pour décrire rupture, dramatisation, événement, habitude.

Le Temps de la Narration dans le récit de L'Etranger est souvent identique avec le Temps de l'Histoire ou de la Fiction, excepté les quelques mini-récits évoquant de souvenirs ; ces souvenirs sont souvent exprimés par une seule phrase seulement et fournissent les analepses du récit. Le présent de la narration est égal au présent du narrateur. Lorsque le protagoniste se rappelle son passé, ce temps de la fiction est exprimé par un temps passé. Il utilise tous les discours, voire des senti-discours, et en parlant du discours direct, Roger Quilliot dit que "l'usage constant de discours semi-directs ( "j'ai dit », « il a répondu ») sont à mettre au compte d'un art réfléchi chez l'auteur et d'une totale indifférence à l'art chez le personnage ${ }^{14}$. Dans ce sens, Brisville dit que « le discours n'est pas [...] son mode naturel d'expression [à Meursault]»15.

Brisville considère le récit de Meursault comme un «monologue »16. Cette forme partage les critiques qui optent pour un journal intime écrit par un détenu. S'il faut croire les critiques qui concluent que Meursault vit au présent et une "vie... instinctive »17, alors, il faut accepter l'idée d'un monologue intérieur. Il y a ceux qui disent que le Meursault

13 Alain RABATEL: La construction textuelle du point de vue, Delachaux et Niestlé S.A., Lausanne (Suisse) - Paris, 1998, p. 45.

14 Roger QUILLIOT : La mer et les prisons, Gallimard, 1956, cité par Brian T. FITCH : Narrateur et Narration dans l'Etranger de Camus, analyse d'un fait littéraire, Lettres Modernes 1960 (deuxième édition revue et augmentée en 1968), p. 21.

15 Jean-Claude BRISVILLE : Camus, Paris, Gallimard, 1959, p. 60.

16 Op. cit., p. 62.

17 Op. cit., p. 59. 
passif n'a pas l'air d'un diariste. Dire cela, voudrait dire que nous n'avons pas réussi à séparer les différents Meursault et les différentes voix que cache ce personnage. Car son «je » semble plus un « jeu » de voix, à chaque fois la même, et à chaque fois une autre; le "Je est un autre " (d'Arthur Rimbaud), dans le cas de Meursault, est valable dans le vrai sens des mots. Ce « je » joue le rôle qui lui a donné l'auteur abstrait, il est sa création sans pourtant faire partie de lui. Un «je » volontairement impliqué par l'auteur abstrait, et à travers lequel, ce dernier passe un message. Tout comme cela pourrait se passer avec un récit à la troisième personne ; alors, pourquoi utiliser « je » ? Pour l'effet troublant, peut-être, que cela provoque chez le lecteur, un appel à s'identifier à ce héros - anti-héros, le temps de la lecture, et pour pouvoir mieux l'éliminer dans la réalité. Pour, peut-être, mieux connaître le Personnage et mieux se comporter comme Personne. Meursault est en réalité un « je » porte-voix de son supérieur - le narrateur abstrait, le narrateur-narrant - se soumettant à la volonté de ce dernier.

En reprenant la parole de Brisville lorsqu'il dit que «Meursault prend la voix d'un témoin qui, l'observant de l'extérieur, n'aurait que le souci de le décrire ", nous sommes amenés à croire, finalement, que le Je-Narrateur est en réalité un «il » déguisé, et qu'il se peut même que, ayant en vue la focalisation externe et le récit extra-hétérodiégétique, ce « je » ne serve que pour cacher les différents types de «il». Ce n'est que vers la fin du texte narratif que le «je » devient authentique, lorsqu'il commence à se personnaliser, à réfléchir et à juger.

\section{*** $*$}

Pour ceux qui ne sont toujours pas convaincus que Meursault est un « je » à multiples voix, nous évoquerons une contradiction le concernant. En effet, un lecteur attentionné remarquera que Meursault-Meursault, le simple employé de bureau, est un homme presque aphone et à l'écoute des autres. Néanmoins, c'est toujours lui qui transmet les paroles des autres personnages, en prêtant sa propre voix. Aussi, Meursault semble-t-il déguiser une idée ou un message à 
Le « Je » polyphonique du monologue intérieur dans

passer de la part d'une personne anonyme derrière le narrateur initial, qui, à son tour, cacherait un narrateur invisible, qui, lui-même cacherait un auteur implicite, puis un auteur explicite, et ainsi de suite. 


\section{La Voix Narrative dans L'Etranger}

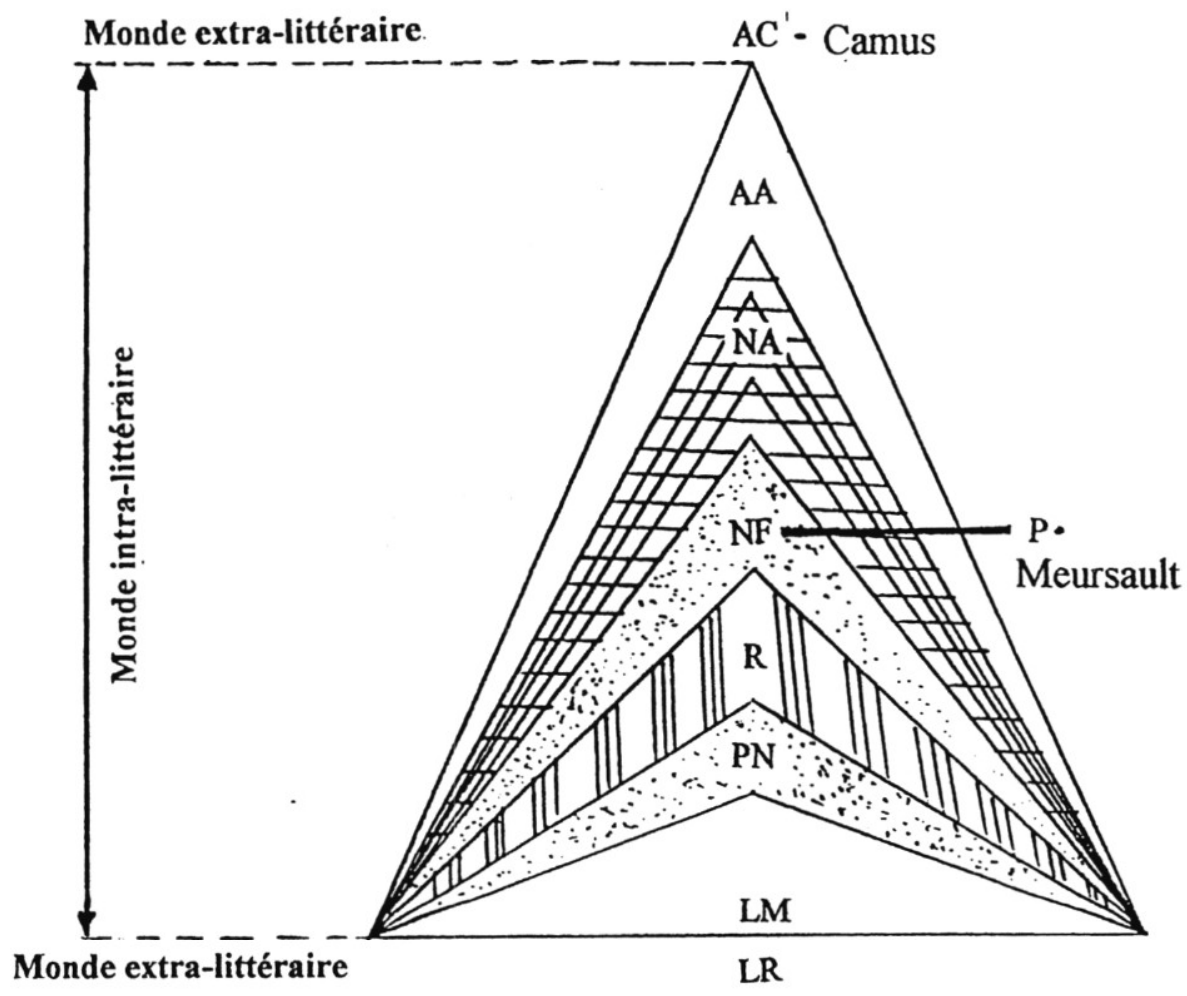

légende

AC - auteur concret

AA - auteur abstrait

NA - narrateur abstrait (N-narrant)

$\mathrm{NF}$ - narrateur fictif (N-narré)

$\mathbf{R}$ - récit

LM - lecteur modèle (narrataire abstrait)

LR - lecteur réel (narrataire concret)

$P$ - personnage

PN - personnage-narrateur 


\section{BIBLIOGRAPHIE}

BRISVILLE (Jean-Claude) : Camus, Paris, Gallimard, 1959.

CAMUS (Albert) : L'Etranger, Paris, Folio-Gallimard, 1942.

FITCH (Brian T.) : Narrateur et narration dans L'Etranger de Camus, analyse d'un fait littéraire, Paris, Lettres Modernes, 1960.

GENETTE (Gérard) : Nouveau discours du récit, Paris, Éditions du Seuil, 1983.

LINTVELT (Jaap) : Essais de typologies narratives, Paris, éd. José Corti, 1981.

NICOLAS (André) : Camus, Paris, 1966 by Pierre Seghers Editeur.

PINGAUD (Bernard) : commente L'Etranger, Paris, FolioGallimard, 1992.

QUILLIOT (Roger) : La mer et les prisons, Paris, Gallimard, 1956.

RABATEL (Alain): La construction textuelle du point de vue, Lausanne-Paris, Delachaux et Niestlé S.A., 1998.

RABATÉ (Dominique) : Vers une littérature de l'épuisement, Paris, éd. José Corti, 1991.

TODOROV (Tzvétan): Les catégories du récit littéraire, in Communications, 8, L'analyse structurale du récit, Paris, Point-Essais, 1966. 